\title{
A SUSPENDED MICROCHANNEL WITH INTEGRATED TEMPERATURE SENSORS FOR HIGH-PRESSURE FLOW STUDIES
}

\author{
S. Wu ${ }^{1}$, J. $\mathrm{Mai}^{2}$, Y. Zohar ${ }^{3}$, Y.C. Tai ${ }^{1}$, and C.M. $\mathrm{Ho}^{2}$ \\ ${ }^{1}$ Electrical Engineering Department, California Institute of Technology \\ ${ }^{2}$ Mechanical \& Aerospace Engineering Department, University of California, Los Angeles \\ ${ }^{3}$ Department of Mechanical Engineering, Hong Kong University of Science \& Technology \\ contact address: S. Wu, Dept., Caltech, Pasadena, CA USA, \\ Email: shuyun@touch.caltech.edu, Fax: (626) 584-9104
}

\begin{abstract}
A freestanding microchannel, with integrated temperature sensors, has been developed for highpressure flow studies. These microchannels are approximately $20 \mu \mathrm{m} \times 2 \mu \mathrm{m} \times 4400 \mu \mathrm{m}$, and are suspended above $80 \mu \mathrm{m}$ deep cavities, bulk micromachined using $\mathrm{BrF}_{3}$ dry etch. The calibration of the lightly boron-doped thermistor-type sensors shows that the resistance sensitivity of these integrated sensors is parabolic with respect to temperature and linear with respect to pressure. Volumetric flow rates of $\mathrm{N}_{2}$ in the microchannel were measured at inlet pressures up to 578 psig. The discrepancy between the data and theory results from the flow acceleration in a channel, the nonparabolic velocity profile, and the bulging of the channel. Bulging effects were evaluated by using incompressible water flow measurements, which also measures $1.045 \times 10^{-3} \mathrm{~N}-\mathrm{s} / \mathrm{m}^{2}$ for the viscosity of DI water. The temperature data from sensors on the channel shows the heating of the channel due to the friction generated by the high-pressure flow inside.
\end{abstract}

\section{INTRODUCTION}

Micromachining is a rapidly emerging technology in which micron-scaled fluidic devices are possible for the fist time. For example microchannels may be used in a variety of applications such as for cooling of electronic circuits, and transport of gases in micro chromatography, for microfluidic control. In order to design and fabricate such micro devices effectively, the physical laws governing fluid flow in small conduits need to be understood. Such flow differs from its macroscopic counterpart in that non-continuum effect for gas or polar mechanics effect for liquid may not be negligible due to the small scale of the flow.

The field of micromachining is still new, and thus it is that only in recent years microchannel flow has attracted considerable attention. Pfahler et al [1] estimated experimentally the friction factor for gas and liquid flow in small channels. Nevertheless Arkilic et al [2] considered the effect of compressibility in a microchannel flow. The most detailed studies up-todate were conducted by Pong, et al. [3] and Shih et al [4] who measured not only the overall pressure drop and flow rate, but also the pressure distribution along the microchannel. However, they were able to obtain data for relatively low inlet pressures beacose of the low operation prassure in the fist generation devices [5]. They showed that by considering slip and compressible effects, the flow rate can be accurately determined.

There are only a few theoretical studies addressing compressible, viscous, steady laminar flow in small uniform conduits. Prud'homme et al. [6] calculated the mass flow rate of gas through a long tube for an ideal gas. Van den Berg [7] presented a detailed study using perturbation methods to calculate the mass flow rate of gas through a cylindrical capillary. They clearly identified the effects of compressibility, acceleration and non-parabolicity of the velocity profile. Harley et al. [8] attempted to analyze the same flow in a microchannel, but as they stated, their model is mathematically inconsistent. Here we present an experimental study of gas and liquid flow through a microchannel under high inlet pressures. We compare the results with an analytical model developed for capillary flow that accounts for 2-D, slip and compressibility and corrected for flow acceleration and non-parabolicity. We show that under very high pressures, the resulting change in channel geometry needs to be considered.

Data for liquid flow through a microchannel is scare since not only a much higher pressure is required to obtain measurable values but also the channels are easily blocked by particles. This project then shows robust design of the present microchannels together with the careful set-up of the external flow handling systems allow us to carry out accurate measurement of a liquid flow rate under high inlet pressure. 
In addition, several investigations have been done to study heat transfer in microchannels [8], but no experimental data on the temperature distribution along a microchannel is available. This information is required to understand the heat transfer process and to develop a complete se of analytical models. This is the motivation behind the development of a suspended microchannel with integrated temperature sensors.

\section{FABRICATION PROCESS}

The freestanding microchannels with integrated thermal sensors were designed and fabricated at Caltech Micromachining Lab. The channel itself was fabricated using surface micromachining techniques, while the thermal isolation cavity and inlet and outlet were bulk micromachined.

The fabrication steps necessary to produce a free standing microchannel are as follows: (1) Starting with a bare $\langle 100\rangle$ oriented silicon wafer, a $1 \mu \mathrm{m}$ thick layer of nitride is deposited via low pressure chemical vapor deposition (LPCVD) and the channel inlet and outlet is patterned. (2) A $2 \mu \mathrm{m}$ LPCVD PSG sacrificial layer, which defines the microchannel length, width, and height, is deposited on the topside of the wafer. (3) A $0.5 \mu \mathrm{m}$ thick layer of LPCVD nitride is deposited to define the channel boundaries. (4) A $0.5 \mu \mathrm{m}$ layer of LPCVD polysilicon is deposited and patterned to form the temperature sensors on top of the microchannel. (5) Selective boron ion implantation is used to control the electrical properties of the polysilicon. The sensor element is $4 \mu \mathrm{m} \times 4 \mu \mathrm{m}$ and is lightly doped with boron $\left(5 \times 10^{18} / \mathrm{cm}^{3}\right)$, giving the sensor a nominal room temperature resistance of $30 \mathrm{k} \Omega$. The poly contact lines to the sensor are heavily boron doped $\left(5 \times 10^{20} / \mathrm{cm}^{3}\right)$. (6) An RIE is performed to open holes through the nitride to the PSG in order to facilitate etching. (7) A $49 \% \mathrm{HF}$ solution is used to etch the PSG in order to sacrificially etch the microchannels. (8) The newly formed microchannel is resealed by uniformly depositing a $0.5 \mu \mathrm{m}$ layer of LPCVD nitride. Microchannel fabrication is now complete with the channel having $1 \mu \mathrm{m}$ thick walls. (9) An anisotropic TMAH solution is used to bulk etch the channel inlet and outlet. (10) Another RIE step is now used to open contacts through the nitride to the polysilicon underneath. (11) Metalization forms bonding pads for connections between the macro world and the sensor elements. (12) Finally, thermal isolation of the channel is achieved by isotropically bulk micromachining the silicon underneath the channel. A photoresist mask is used to protect the channel and its suspension stringers from a $\mathrm{BrF}_{3}$ dry etch [9], which leaves a cavity $80 \mu \mathrm{m}$ deep and about $500 \mu \mathrm{m}$ wide.
The channel used in all the following experiments documented in this paper is $4.4 \mathrm{~mm}$ long, $1.85 \mu \mathrm{m}$ in heigh, and $19 \mu \mathrm{m}$ in width. These dimensions were obtained using an Alpha step profiler after the test channel ruptured. The channel is suspended by 200 $\mu \mathrm{m}$ long stringers on each side, which are spaced approximately $250 \mu \mathrm{m}$ apart. The temperature sensor contact lines run from the center of the channel, out along the stringers, to bonding pads next to the isolation cavity

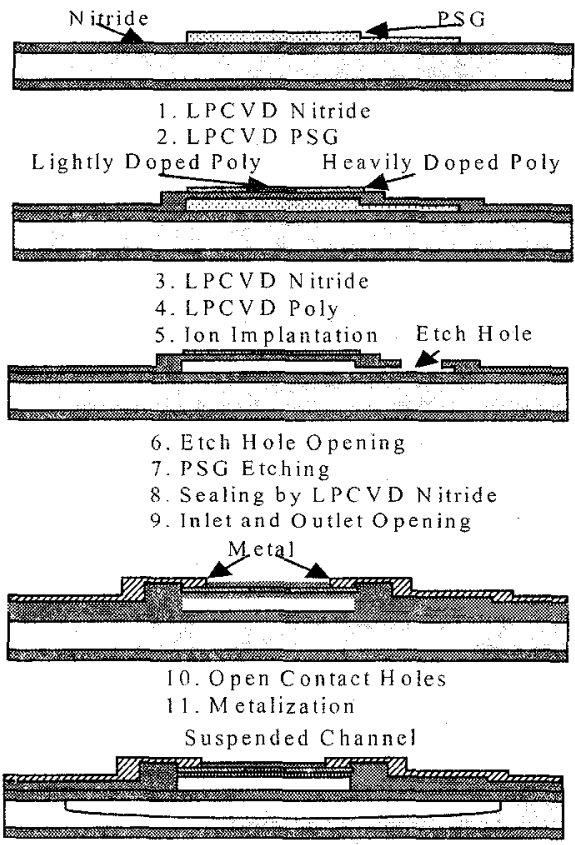

12. Si Undercutting by BrF

Fig. 1 Microchannel process flow

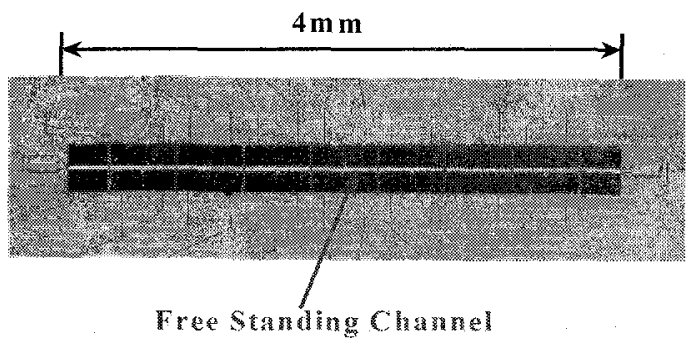

Fig. 2 Photograph of the top view of a free standing microchannel.

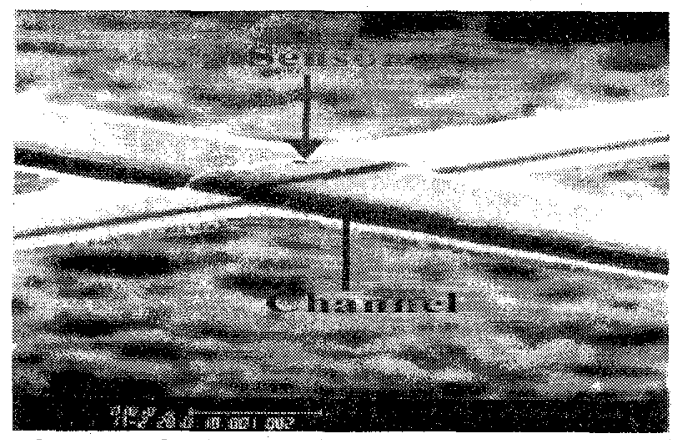

Fig. 3 SEM of a freestanding channel with a temperature sensor embedded onto the top wall

TEST PROCEDURE 
All experiments were setup and conducted at Caltech. Gas and liquid flow measurements were conducted in the apparatus illustrated in Figure 4. Microelectronic grade $\mathrm{N}_{2}$ from a pressurized canister was passed through HPLC tubing into the suspended microchannel. An Omega type PX120 pressure transducer was used to monitor the inlet pressure. An Omega model HHP3200 digital manometer measured the exit pressure. These transducers had an uncertainty of 1.0 and 0.1 psia, respectively. The volume flow rate was measured using various glass tubes approximately 1.1 meter in length, and from $3 \mathrm{~mm}$ to $8 \mathrm{~mm}$ in (outer) diameter, which lever was appropriate for the flow rate being investigated. These tubes were calibrated using water and a graduated cylinder. The volume flow rate in the tube was measured optically as a meniscus of water traveled past marked gradations versus time. Data for each set of flow parameters (at various inlet and outlet pressures) took from thirty minutes (for a gas) to four hours (for a liquid), depending on the relative flow rate. In addition, a vacuum/compressor pump is connected to the settling chamber at the end of the measurement tube. This allows the exit pressure to be lowered to a maximum vacuum of $-12 \mathrm{psig}$, or pressurized to 20 psig. However, in order to truly ascertain the exit pressure, it is necessary to integrate a pressure transducer immediately before the exit of the microchannel. This transducer will be integrated into the next generation of suspended microchannels.

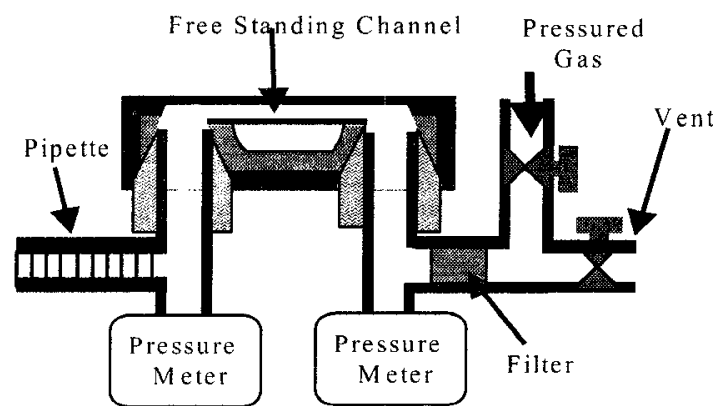

Fig. 4 illustration of the microchannel and flow measurement system.

Temperature calibrations of the embedded sensors were conducted inside a Delta Design Model 2300 isothermal oven. Resistances were measured by a HP Model 34401A multimeter, and accurate temperature measurements, better than $0.01{ }^{\circ} \mathrm{C}$, were obtained using an Omega DP-251 precision thermometer using a calibrated RTD sensor element.

The temperature distribution along the pressurized channel was measured in a vacuum chamber, The chamber was evacuated to $10 \mathrm{mtorr}$ in order to minimize heat loss due to convection effects.

\section{Sensor Calibration}

Since this microchannel is intended for high-pressure heat transfer experiments, the sensor's sensitivity due to temperature and pressure must be characterized. Lightly doped polysilicon displays piezoresistive properties; therefore, it is important to not only calibrate the sensor's resistive output as a function of temperature, but also compensate for any cross talk due to pressure. The data shows that the sensor's temperature sensitivity is independent of pressure in the tested range from 0 to 300 psig. Figure 5 displays the nonlinear resistance dependence on temperature of three embedded sensors. Furthermore, the curves collapse together, showing the uniformity of their sensitivity to temperature. Similarly, Figure 6 shows the sensor resistance change due to variations in pressure. The resistance sensitivity of the sensor to pressure is linear in the test range, and is independent of temperature in the range from $10^{\circ} \mathrm{C}$ to $40^{\circ} \mathrm{C}$. Thus, the sensor's sensitivity is parabolic to temperature, and is linear to pressure, can be expressed as

$$
\left(\mathrm{R}-\mathrm{R}_{\mathrm{o}}\right) / \mathrm{R}_{\mathrm{o}}=\alpha_{2}\left(\mathrm{~T}-\mathrm{T}_{\mathrm{o}}\right)^{2}+\alpha_{1}\left(\mathrm{~T}-\mathrm{T}_{\mathrm{o}}\right)+\beta\left(\mathrm{P}-\mathrm{P}_{\mathrm{o}}\right)
$$

where $\alpha_{1}, \alpha_{2}$, and $\beta$ are experimentally determined to be approximately equal to $0.009{ }^{\circ} \mathrm{C}^{-1}, 6 \times 10^{-5}{ }^{\circ} \mathrm{C}^{-2}$, and $10^{-5} \mathrm{psi}^{-1}$, respectively.

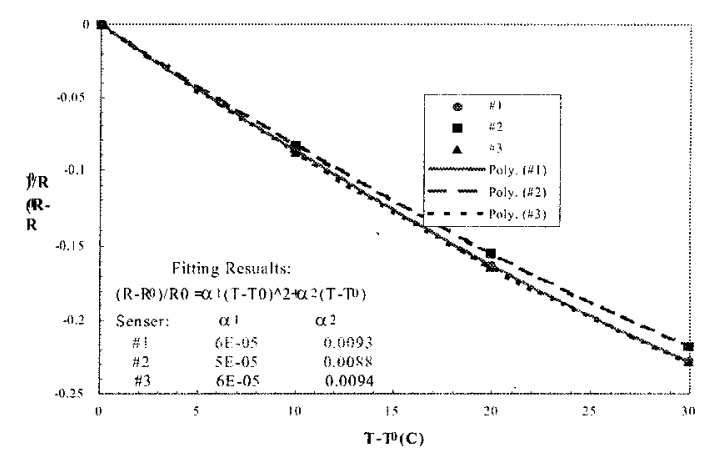

Fig. 5. Sensor sensitivity to temperature.

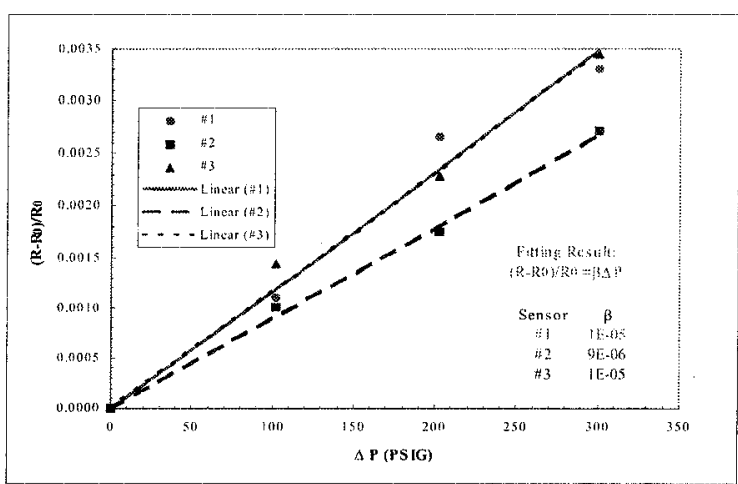

Fig, 6 Sensor Sensitivity to pressure

RESULTS AND DISCUSSION 


\section{Fluid Flow}

All classical analysis of channel flow begins with the momentum equation for a compressible, steady, twodimensional flow

$$
\rho u \frac{\partial u}{\partial x}=-\frac{\partial u}{\partial x}+\mu\left(\frac{\partial^{2} u}{\partial^{2} y}+\frac{\partial^{2} u}{\partial^{2} x}\right)
$$

\section{Liquid Flow Rate Measurements}

Before moving onto the analysis of flow, we investigate the behavior of liquids, since even at elevated pressures, the behavior of liquids is still incompressible. The data for DI water flow in the microchannel is expected to be linear with pressure according to the mass flow rate equation for incompressible channel flow is

$$
Q_{v}=\frac{h^{3} w}{12 \mu L} \Delta P
$$

where DP is the pressure difference between the inlet and outlet Figure 7 displays data for the volume flow rate for DI water in the channel at various inlet pressures, which is nonlinear. This suggests that the channel deforms under high pressure since it deviates from the theoretical values calculated using Eq. 3, as illustrated by the dashed line in Figure 8 . We investigate this assumption, by modifying the height term in the mass flow equation to include the effect of high-pressure deflection:

$$
h=h_{o}\left(1+\alpha P_{L}\right)
$$

where $h_{o}$ is the nominal height of $1.85 \mu \mathrm{m}$ at standard conditions and $P_{L}$ is the local pressure. Assuming that $\alpha P_{L}$ is small so that higher order terms can be ignored, this yields the following approximation for volume flow

$$
Q_{v}=\frac{h_{0}{ }^{3} w}{12 \mu L} \Delta P\left(1+\frac{3}{2} \alpha \Delta P\right)
$$

It is clear that this geometry correction is parabolic with respect to the pressure difference and explains the curvature of the data as seen in Figure 8 . By fitting the experimental data with Eq. 5 , this yields $\mu=1.045 \times 10^{3}$ $\mathrm{N}-\mathrm{s} / \mathrm{m}^{2}$ and $\alpha=0.000264 \mathrm{psi}^{-1}$. The reliability of the method can be seen by the fact that our estimated viscosity is consistent with the published value by taking into account the experiment error (less than 5\%). The effective deflection experienced at 500 psig for each channel wall should be approx. $0.7 \%$.of width, or $7 \%$ of height., which correspond to 0.13 micron. Thus, in future channel design, if we can reduce the bulging by designing channels with thicker walls, we can then concentrate our studies on the flow properties such as viscosity, compressibility, slip boundary or even more complicated effects like acceleration and non-parabolic velocity profile under high pressure.

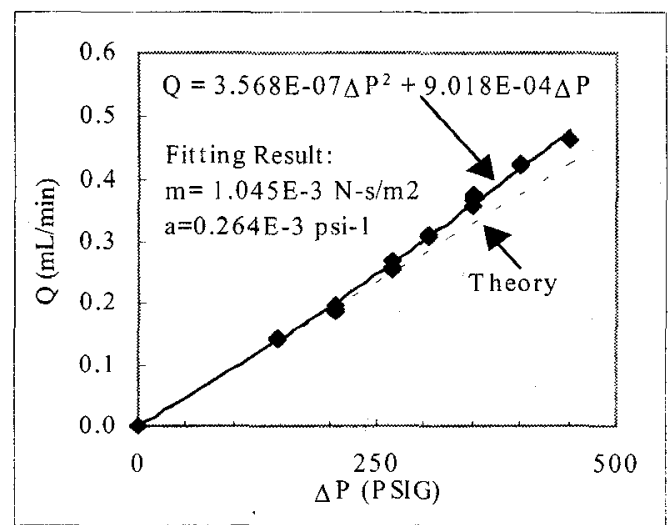

Fig. 7 Volume flow rate vs. inlet pressure for an incompressible liquid.

\section{Low-pressure gas flow}

In our situation, nitrogen gas was passed through the channel at low and high pressures. We now define low pressure as being under $60 \mathrm{psia}$, which is comparable to the pressure range already investigated $[2,3,4,8]$. By assuming a parabolic velocity profile and neglecting the convection term, the mass flow rate for a compressible flow with slip is expressed in the following form, as derived from equation 1 .

$Q_{m}=\frac{h_{0}{ }^{3} w P_{o u t}^{2}}{24 R T L \mu}\left[\left(\left(\frac{P_{i n t}}{P_{\text {out }}}\right)^{2}-1\right)+12 K n_{\text {out }}\left(\frac{P_{\text {in }}}{P_{\text {out }}}-1\right)\right]$

where $\mathrm{Kn}$ is the Knudsen number at the exit of the channel, $\mathrm{R}$ is the appropriate gas constant, and $\mu$ is the dynamic viscosity of the gas.

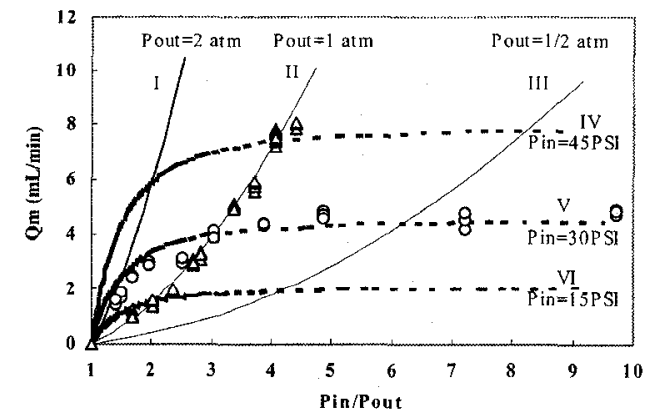

Fig. 7 Theory curves and experimental data for low pressure $\mathrm{N}_{2}$ flow.

In Figure 7 two sets of curves are plotted. Curve II is the standard method of illustrating Eq. 6 , where $P_{\text {out }}$ is 
constant and chosen to be 1 atmosphere. However, if $\mathrm{P}_{\text {out }}$ is kept at a different constant pressure, curves I and III are sample results. Travel right to left from curve III to I, the fixed outlet pressure changes from $2 \mathrm{~atm}$. to 0.5 atm. Then curves IV, V, and VI, also calculated using Eq. 3, allow comparison of trends with fixed inlet conditions. Experimental data at a fixed outlet pressure of $1 \mathrm{~atm}$. is compared to curve II. Also, data at a fixed inlet pressure of 30 psig is compared to curve IV. Within experimental error, these data points, which are obtained by applying appropriate pressure or vacuum at the exit in order to generate the desired pressure, fit these generally accepted low pressure theoretical predictions. The consistency between the data and theoretical calculations show that the experimental setup provides an appropriate method to obtain extremely small volume flow rate measurements. Further, these results support the argument that flow acceleration and non-parabolicity effects are negligible at low pressures.

\section{High-pressure Gas Flow}

In order to estimate the influence of the flow terms ignored so far in this analysis, we refer to the work of van den Berg, et al.[5] for guidance. They derived equations for mass flow rate which takes into consideration the non-parabolicity of the velocity profile, as wells as the convection (acceleration) term of the momentum equation. However, their analytical study was for a capillary with a circular cross-section. Using hydraulic diameter arguments, we assume that the relative contributions of these effects for pipe flow are on the same order of magnitude for channel flow. Therefore, the factors contributing to the total mass flow rate can be estimated as follows

$$
Q_{m}=Q_{m i}\left[1+\eta_{c}+\eta_{s}\right]\left(1-\eta_{a}\right)\left(1-\eta_{p}\right)
$$

where the incompressible mass flow rate is equal to the volume flow rate in $\mathrm{Eq} 4$., multiplied by the average density of the gas with respect to the average pressure. Hence the compressibility correction is expressed as

$$
\eta_{c}=\frac{1}{2}\left(\frac{P_{\text {in }}}{P_{\text {out }}}-1\right)
$$

the correction due to slip effects is

$$
\eta_{\mathrm{s}}=6 \mathrm{Kn}
$$

the contribution from the convection term is

$$
\eta_{a}=\frac{Q_{m i}}{6 \pi \mu L} \ln \frac{P_{i n}}{P_{o u t}}
$$

and the contribution of a non-parabolic velocity profile has an effect on the order of

$$
\eta_{p}=\frac{Q_{m i}}{12 \pi \mu L} \ln \frac{P_{\text {in }}}{P_{\text {out }}}
$$

Substituting in values, for $\eta_{\mathrm{a}}$ and $\eta_{\mathrm{p}}$, it is apparent that these terms are much less than 1 , so higher order terms can be neglected and Eq. 7 can be rewritten as

$Q_{m}=Q_{m i}\left[1+\eta_{c}+\eta_{s}\right]\left(1-\eta_{a}-\eta_{p}\right)$

It is readily apparent that when the acceleration effect and the effect due to the non-parabolic velocity profile are taken into consideration, this should actually result in a lower theoretical mass flow rate. For example, the difference between the usual non-slip curve and the curve with takes into account acceleration and nonparabolicity is about $6 \%$ at 600 psig (pressure ratio is about 40), as seen in Figure 9.

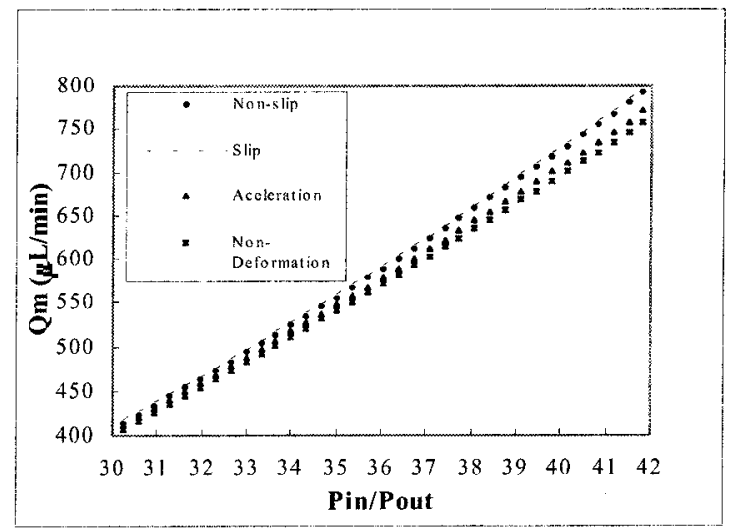

Fig. 9 Mass flow rate vs. inlet pressures, with curves at various exit pressures, slip and no slip theoretical curves, and a curve with acceleration effects.

In Figure 9, at higher-pressure ratios the data deviates from the lower curve, which takes into consideration acceleration and a non-parabolic velocity profile. This discrepancy between measured and theoretical values calculated from Eq. 12 can now only be attributed to deformations in the channel geometry due to high pressure of the channel. An effective mass flow rate can be written as Eq. 13,

$$
Q_{\text {effective }}=Q_{m}\left(1+\eta_{\text {def }}\right)
$$

which takes into consideration flow acceleration and non-parabolicity, as well as geometry. In Eq 13, the deformation correction term is given by

$$
\eta_{\text {def }}=\alpha P_{i n}\left[1-\left(\frac{P_{o u t}}{P_{i n}}\right)^{3}\right]
$$

which is derived from the gas flow momentum equation by using Eq 5 again to model the high-pressure deflection 
Figure 10 illustrates that the measured data fits the mass flow rate theory when flow acceleration, non-parabolic flow velocity profile, and channel deformation due to high pressures is taken into consideration.

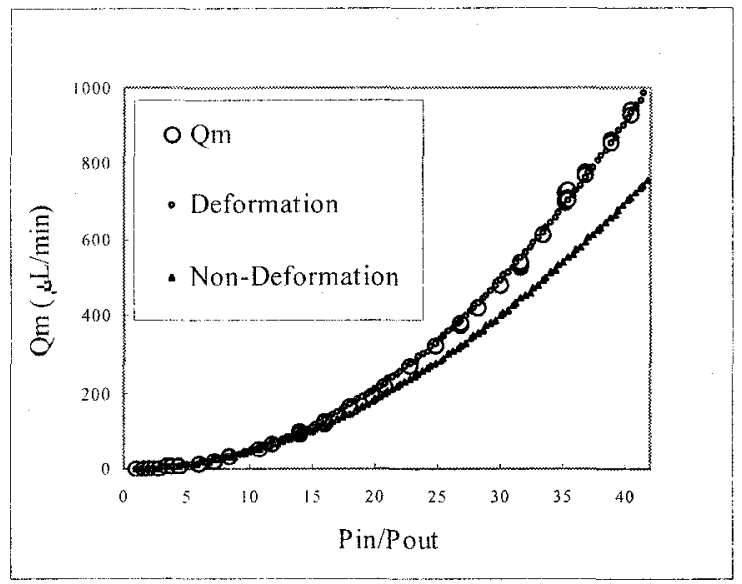

Fig. 10 Mass flow rate vs inlet pressure.

\section{Temperature Measurement}

Finally, data relating to heating of the channel, due to friction generated by the flow, has been obtained, as graphed in Figure 11. The entire device was thermally isolated in a vacuum, and then the channel pressurized to either 271 or 305 psig. The data was recorded from 3 sensors as the flow was reversed between inlet and outlet. All three sensors indicate lower temperature than the ambient. Furthermore, the inner sensor shows higher temperature than that of the two outer sensors. This might indicate that there is some heat loss at the edges through conduction via the coupling.

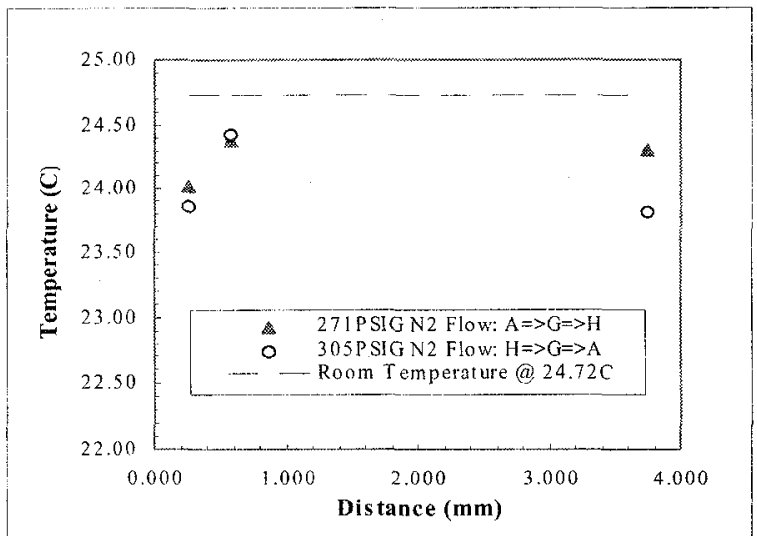

Fig. 11 Temperature distribution along the channel with the N2 flow forced by 300 psig.

\section{CONCLUSION}

The robust nature of these microchannels facilitated high-pressure measurements. Mass flow data at unprecedented high inlet pressures has been successfully obtained, and the deviation of this data from theory (used to obtain Eqs.11 and 10) is attributed to flow effects such as local flow acceleration and a non-parabolic velocity profile, as well as to bulging in the channel. This bulging effect was isolated and measured using incompressible liquid flow. In addition, at a fixed inlet pressure, a complete saturation flow rate curve has been obtained by evacuating and pressurizing the exit of the channel, however, choke at higher inlet pressures can not be conclusively determined until we integrate pressure sensors at the outlet of the next generation channel. Most importantly, we obtained coefficients for the parabolic dependence of the resistance of the imbedded thermal sensors to temperature, as well as the coefficient for the sensor's linear dependence on pressure. The resulting temperature data implies the effect of friction along the channel.

\section{ACKNOWLEDGEMENTS}

This work is sponsored by the Air Force Office of Scientific Research, USAF, under grant/contract number 49620-96-1-0376. The authors would like to thank Trevor Roper, Tom Tsao and Xuan-Qi Wang for the help with the process.

\section{REFERENCES}

[1] J. Pfahler, J. Harley, H. Bau, J. Zemel, "Gas and liquid flow in small channels," Symp. Micromechanical Sensors, Actuators, and Systems (ed. D. Cho, R. Warrington, et al.) ASME DSC v.32, p49-60, 1991. [2] E.B. Arkilic, M.A. Schmidt, K.S. Breuer, "Gaseous flow in microchannels," Application of Microfabrication to Fluid Mechanics, ASME Winter Annual Meeting, pg. 57-65, Chicago, IL Nov. 1994. [3] K.C. Pong, C.M. Ho, J. Liu, Y.C. Tai, "Non-linear pressure distribution in uniform microchannels," Application of Microfabrication to Fluid Mechanics, ASME Winter Annual Meeting, pg. 51-56, Chicago, IL Nov. 1994.

[4] J. Liu, Y. C. Tai, K. C. Pong, and C. M. Ho, "Micromachine channel/pressure sensor systemf for "microflow studies", Transducers' 93, pp.995-997 [5] J.C. Shih, C.M. Ho, J. Liu, Y.C. Tai, "Monatomic and polyatomic gas flow through uniform microchannels," Application of Microfabrication to Fluid Mechanics, ASME Winter Annual Meeting, pg. 197-203, Atlanta, Ga, Nov. 1996.

[6] R.K. Prud'homme, T.W. Chapman, J.R. Bowen, "Laminar compressible flow in a tube," Appl. Sci. Res. v.43, p.67-74, 1986.

[7] H.R. van den Berg, C.A. Seldam, P.S. Gulik, "Compressible laminar flow in a capillary," J.Fluid Mechanics, v. 246, p.1-20, 1993.

[8] J.C. Harley, Y. Huang, H.H. Bau, J.N. Zemel, "Gas flow in micro-channels," J.Fluid Mechanics, v. 284, pp257-274, 1995.

[9] X. Wang, X. Yang and Y. C. Tai, " Gas-Phase Silicon Etching with Bromine Trifluoride, pp.2D3.02, Transducer '97, Chicago 